\title{
Development of trigger and start detectors for experiments with high-energy heavy ions at the Joint Institute for Nuclear Research
}

\author{
V. I. Yurevich*, G. N. Agakichiev, S. V. Sergeev, D. N. Bogoslovski, S. P. Lobastov, \\ V. Yu. Rogov, G. S. Averichev, V. V. Tikhomirov and A. A. Timoshenko \\ Laboratory of High Energy Physics, Joint Institute for Nuclear Research \\ Dubna, Moscow region 141980, Russian Federation \\ yurevich@jinr.ru \\ O. I. Batenkov \\ Radium Institute \\ St. Petersburg 194021, Russian Federation
}

Published 10 July 2018

\begin{abstract}
Fast Cherenkov and scintillation detectors based on microchannel-plate photomultiplier tubes and silicon photomultipliers are being developed for application in the multi-purpose detector and baryonic matter at the Nuclotron experiments with the heavy ion beams at the Joint Institute for Nuclear Research. The aim of the detectors is the fast triggering of nucleus-nucleus collisions with high efficiency and to generate the start signal with picosecond time resolution for time-offlight measurements. The detectors provide a time resolution better than $50 \mathrm{ps}$ and can operate in the strong magnetic field of the experimental facilities.
\end{abstract}

Keywords: Nucleus-nucleus collisions; interaction trigger; T0 detector; picosecond time resolution.

PACS numbers: 29.40.Ka, 29.40.Gx

\section{Introduction}

The efficient selection of nucleus-nucleus collision events by an L0 trigger is a key challenge in collider and fixed-target experiments with high-energy beams of heavy ions. Another important problem is the generation of the start signal (T0) pulse with picosecond time resolution for time-of-flight (TOF) detectors, because the quality of charged-particle identification highly depends on the time resolution of the TOF system. Operation in the strong magnetic field typical of an experimental setup is another usual requirement to detectors in these systems.

The fast triggering of nucleus-nucleus collisions and a precise TOF measurement with picosecond time resolution are important features of all experiments at the Relativistic Heavy Ion Collider (RHIC) and the Large Hadron Collider (LHC). For these aims, a two-arm modular detector with fast Cherenkov or scintillation counters is used.

This is an Open Access article published by World Scientific Publishing Company. It is distributed under the terms of the Creative Commons Attribution 4.0 (CC-BY) License. Further distribution of this work is permitted, provided the original work is properly cited. 


\section{I. Yurevich et al.}

Our group is responsible for developing the fast interaction trigger and T0 detectors for the future multi-purpose detector $(\mathrm{MPD})^{1}$ collider experiment and the fixed-target study of baryonic matter at the Nuclotron $(\mathrm{BM} @ \mathrm{~N})^{2}$ experiment. The goal of these experiments is to study nuclear matter properties at the extreme conditions produced in nucleus-nucleus collisions at the energies reached at the Nuclotron-based Ion Collider facility (NICA) ${ }^{3}$ at the Joint Institute for Nuclear Research (JINR) in Dubna, Russia.

\section{Fast Forward Detector for MPD Experiment}

NICA will operate at much lower beam energy (from 0.6 to $4.5 \mathrm{AGeV}$ ) than the RHIC and LHC. It leads to a rather low multiplicity of particles produced in heavy-ion collisions, and those particles are not ultra-relativistic (even the spectator protons). As a result, the effective triggering of $\mathrm{Au}-\mathrm{Au}$ collisions and the picosecond time resolution of start signal T0 are two nontrivial problems which are solved by the development of the fast forward detector (FFD) in the MPD project. The requirements of the FFD are: (i) the triggering of central and semi-central $\mathrm{Au}-\mathrm{Au}$ collisions with $100 \%$ efficiency, (ii) a time resolution of start pulse $\sigma_{\mathrm{T} 0}<50 \mathrm{ps}$, (iii) trigger dead time of less than $75 \mathrm{~ns}$, and (iv) operation in the MPD's magnetic field of $B=0.5 \mathrm{~T}$.

To reach the above requirements, we developed two identical modular sub-detectors, $\mathrm{FFD}_{\mathrm{E}}$ and $\mathrm{FFD}_{\mathrm{W}}$, with large active areas placed close to the beam vacuum pipe at a distance of $140 \mathrm{~cm}$ to the left and right, measured from the center of the MPD setup. The FFD covers the pseudo-rapidity interval of $2.7<|\eta|<4.1$ and it provides an efficient trigger for $\mathrm{Au}-\mathrm{Au}$ collisions by detecting photons from $\pi^{0}$ decays and relativistic charged particles, as shown in Fig. 1.

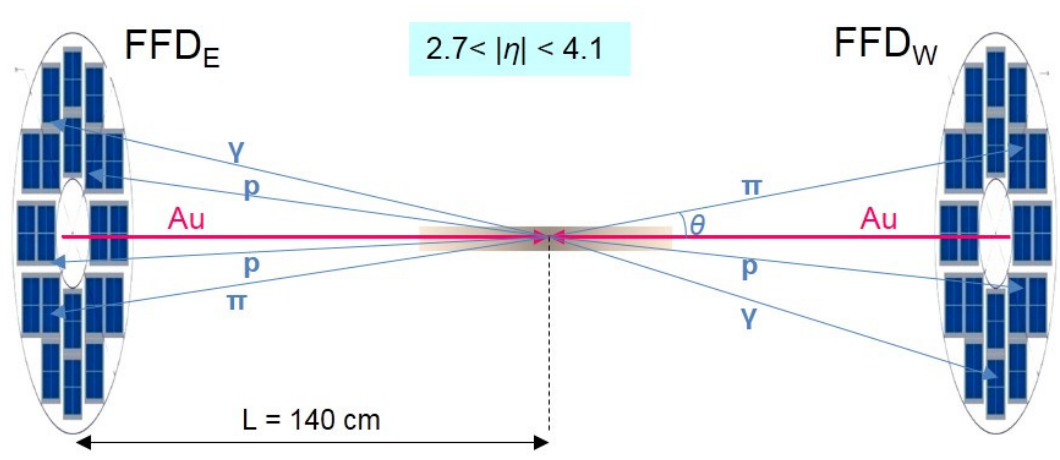

Fig. 1. A schematic view of the FFD.

Each FFD array consists of 20 modules with total granularity of 80 channels, plus 20 channels with common pulses of the modules. The inner diameter of the modular array is approximately $90 \mathrm{~mm}$; the outer diameter is approximately $360 \mathrm{~mm}$. 
The study of FFD performance was carried out by Monte Carlo simulation with QGSM + GEANT4 code. It was shown that detection of high-energy photons from $\pi^{0}$ decays can improve the trigger efficiency and the time resolution of the detector. In FFD modules, the high-energy photons are efficiently detected by conversion to electrons in a lead plate. The electrons leaving the lead plate produce Cherenkov light in a quartz radiator connected to a photodetector. A multi-anode microchannel-plate photomultiplier tube (MCP-PMT), the XP85012/A1-Q from Photonis, is used to detect the Cherenkov photons. It is suitable for designing large modular arrays with little dead space, providing excellent time resolution and magnetic field immunity.

The FFD module has a two-by-two cell design and includes a lead converter, a Cherenkov radiator with four quartz bars, $28 \times 28 \times 15 \mathrm{~mm}^{3}$ each, an MCP-PMT XP85012/A1-Q photodetector, and a front-end electronics (FEE) board. The XP85012/A1 with 64 anode pads is transformed into a four-channel photodetector by merging 16 pads $(4 \times 4)$ into a single channel of FEE. In addition to the anode pad signals, this device has a common MCP output. The time resolution obtained with this signal is slightly lower than the resolution obtained with the anode signal. Pulses from the MCP's outputs are used to determine interaction point position by a fast vertex algorithm, the signal of which is used to generate the interaction trigger pulse. Also, these pulses are useful for monitoring FFD operation. Thus, the FEE of each FFD module has five independent channels (four individual and one common) producing analog and lowvoltage differential signaling (LVDS) pulses.

The efficiency of photon registration depends on converter thickness and photon energy. This was studied using Monte-Carlo simulation to determine the ideal thickness of the lead converter for the photon energy interval covered by the energy spectrum of photons produced in $\mathrm{Au}-\mathrm{Au}$ collisions. Finally, a 10-mm lead converter was chosen.

The efficiency of a fast vertex trigger for $\mathrm{Au}-\mathrm{Au}$ collisions was studied for two cases: (1) ideal sub-detectors without any dead gaps between modules, and (2) real modular arrays of FFD. In both cases, the obtained efficiency is $100 \%$ up to the impact parameter of $11 \mathrm{fm}$ at any energy of the NICA collider. Peripheral collisions are the only causes of decreases in efficiency.

The time resolution of time-of-flight measurements is

$$
\sigma_{t}=\left(\sigma_{T O F}^{2}+\sigma_{T 0}^{2}\right)^{1 / 2}
$$

where $\sigma_{\mathrm{TOF}}$ represents the time resolution of the TOF detector, and $\sigma_{\mathrm{T} 0}$ is the time resolution of the start signal. The value $\sigma_{\mathrm{T} 0}$ is calculated as

$$
\sigma_{T 0}=\left(\sigma_{F F D}^{2}+\Delta t_{f h}^{2}\right)^{1 / 2}
$$

where $\sigma_{\mathrm{FFD}}$ represents the time resolution of the FFD channel studied experimentally, and $\Delta t_{f h}$ is the time uncertainty of the first particle's arrival in the FFD. 
The start signal (T0) for the TOF detector requires the appearance of at least one pulse with good timing characteristics from all FFD channels. These pulses are used for off-line determination of T0, and the time resolution improves by a factor $N^{1 / 2}$, where $N$ is the number of these pulses.

Three different methods of readout were used in the test measurements: (1) an Evaluation Board DRS4 digitizer produced in PSI, (2) a CAEN model N6742 digitizer based on the DRS4 chip, and (3) TDC72VHL VME modules produced by the Laboratory of High Energy Physics, JINR and used in the MPD Data Acquisition System. The first two methods use analog pulses from the FEE of FFD modules; the last method uses LVDS pulses.

The TOF measurements with two modules and a beam of 3.5-GeV deuterons showed that the time resolution of the FFD module itself is $\sigma \approx 21.5 \mathrm{ps}$. The measurements with short cables and different readout electronics gave $\sigma \approx 24$ ps with the DRS4 digitizer, 34 ps with the CAEN digitizer, and 44 ps with TDC72VHL. The TOF peak measured with a full chain of cables, electronics, and the TDC72VHL readout is shown in Fig. 2. It corresponds to the above-mentioned time resolution of $\sigma \approx 44 \mathrm{ps,} \mathrm{which} \mathrm{is} \mathrm{better} \mathrm{than} \mathrm{the}$ 50 ps required.

The detector modules operated well in our tests with the magnetic field of the BM@N magnet operating at up to $B=0.9 \mathrm{~T}$. Some results obtained in the test measurements have been reported and published elsewhere. ${ }^{4,5}$

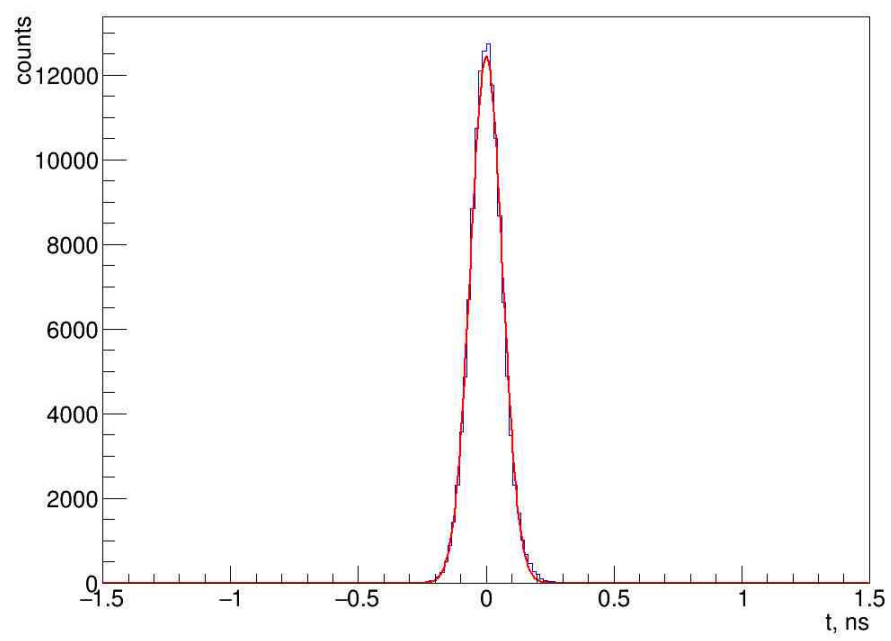

Fig. 2. The TOF peak obtained in measurements with TDC72VHL and a full chain of cables and electronics.

\section{Trigger and T0 System for BM@N Experiment}

In the $\mathrm{BM} @ \mathrm{~N}$ experiment, the beam ion energy range is $1-4.5 A \mathrm{GeV}$, and the type of ions varies from the lightest nuclei to gold. The BM@N setup must be able to operate with a beam intensity of up to $10^{7} \mathrm{Au}$ ions per spill. 
The beam line and target area detector system provides:

- Active transport of HI to a target by upstream detectors.

- Fast and effective triggering $A A$-collisions in a target by target area detectors.

- Generation of a precise start signal with $\sigma_{\mathrm{T} 0}<50$ ps for the TOF detectors.

- Monitoring of beam conditions, interaction rate, beam intensity, and spill characteristics.

As indicated above, the target area detectors must operate in a strong magnetic field of up to $B=0.9 \mathrm{~T}$. To minimize the background interactions of beam ions with beam line materials, all beam detectors must be as thin as possible. For realization of these requirements, a special detector and trigger system was developed.

$\mathrm{A}$ beam counter $\mathrm{BC} 1$ detects an incoming beam ion $\sim 15 \mathrm{~m}$ upstream from a target. $\mathrm{A}$ veto counter $\mathrm{VC}$ has a hole for beam ions and its position is $2.5 \mathrm{~m}$ upstream from the target. It is used for rejection of events with a background of halo particles. The last upstream detector is a beam counter BC2 with a small and thin scintillator and position of $z=20 \mathrm{~cm}$ from the target. Only pulses produced by beam ions are selected by the trigger; pulses with smaller pulse heights are discarded. Also, this counter is used as the T0 detector for runs with beam intensity below $\sim 10^{6}$ ions per spill. For a higher beam intensity, the Cherenkov detector $\mathrm{CD}$ is used as the T0 detector. The last scintillation beam counter BC3, located at $z=12 \mathrm{~cm}$ downstream, is used for the fast selection of events with pulse heights smaller than the pulse heights of beam ions, which correspond to interactions in a target. All these detectors, $\mathrm{BC} 2, \mathrm{BC} 3$, and $\mathrm{CD}$, are based on $\mathrm{MCP}-$ PMTs from Photonis, and they can operate in the magnetic field of BM@N. The Cherenkov detector CD is based on the same modules as the FFD in the MPD project. It consists of two identical arrays, $\mathrm{CD}_{1}$ and $\mathrm{CD}_{2}$, with four modules in each array.

Two different multichannel detectors, a barrel detector (BD), and a silicon detector (SiD) are used for triggering $A A$-collisions on centrality due to a well-known fact: more central collisions produce larger number of particles. For this purpose, a threshold on a number of fired channels in both detectors is set. The BD is based on scintillation strips with a silicon photomultiplier ( $\mathrm{SiPM}$ ) readout and detects secondary charged particles in the region of target nucleus fragmentation. The segmented $\mathrm{SiD}$ has a position of $z=7 \mathrm{~cm}$ behind the target, and it detects charged particles in the forward region.

A scheme of the detector layout in the BM@N target area is shown in Fig. 3. All main trigger and $\mathrm{T} 0$ detectors are located in a compact zone, forming the active target area.

The analog and LVDS pulses from detectors are fed to inputs of a trigger module T0U developed for the BM@N experiment. The module is based on FPGA and performs the fast processing of input signals in correspondence with the programmed trigger logic. Several different trigger logics can be chosen by the user:

1. Valid beam: $\mathrm{VB}=\mathrm{BC} 1 * \mathrm{BC} 2 * \mathrm{VC}_{\text {veto, }}$

2. No collision in target: $\mathrm{NC}=\mathrm{VB} * \mathrm{BC} 3\left(A_{\text {beam }}\right) * \mathrm{BD}_{\text {veto, }}$, 
3. Minimum bias collision: $\mathrm{MBC}=\mathrm{VB} * \mathrm{BC} 3\left(A_{\text {beam }}\right)_{\text {veto }}$, and

4. Central collision: $\mathrm{CC}=\mathrm{MBC} * \mathrm{BD}\left(N>N_{1}\right) * \mathrm{SiD}\left(N>N_{2}\right)$,

where $A_{\text {beam }}$ is the pulse height produced by a beam ion and $N$ is the number of fired channels.

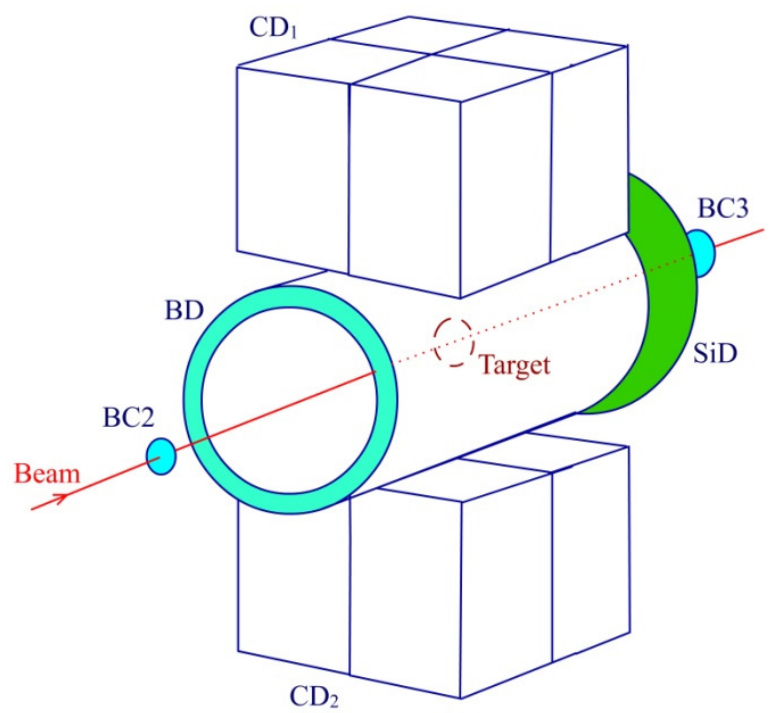

Fig. 3. A scheme of the BM@N target area.

In future runs with maximum beam intensity, the beam counters will be removed and the interaction trigger will be produced by the coincidence of pulses from the BD, $\mathrm{SiD}$, and CD detectors.

The T0U module was successfully tested and was used in BM@N runs from 2015 to 2017 with beams of deuterons and carbon ions. In the March 2017 BM@N run, two versions of the BC2 (T0) detector with the PP0365G MCP-PMT were used to study the time resolution. The first was the Cherenkov detector $\mathrm{BC} 2_{\mathrm{Ch}}$ with $4-\mathrm{mm}$ quartz $\left(46^{\circ}\right.$ to the beam axis) and the second was the scintillation detector $\mathrm{BC} 2_{\mathrm{sc}}$ with a $0.8-\mathrm{mm}$ scintillator $\left(45^{\circ}\right.$ to the beam axis). The MCP-PMT pulses were fed to a FEE board with analog and LVDS outputs. The typical analog pulses of the scintillation detector measured with the CAEN digitizer are shown in Fig. 4. The interaction events were selected in this run with the following trigger logic:

$$
\mathrm{IT}=\mathrm{BC} 1 * \mathrm{BC} 2_{\mathrm{Ch}} * \mathrm{BC} 2_{\mathrm{sc}} * \mathrm{VC}_{\mathrm{veto}} * \mathrm{BD}(N \geq 3) .
$$

A result of a TOF measurement with these two counters and $4.5 \mathrm{AGeV}$ carbon ions is shown in Fig. 5. It demonstrates excellent time resolution for both detectors with 
$\sigma_{\mathrm{T} 0} \approx 30 \mathrm{ps}$ per detector. Thus, the obtained result proves that the $\mathrm{BC} 2$ detector with the thin scintillator can be used for production of a T0 pulse.

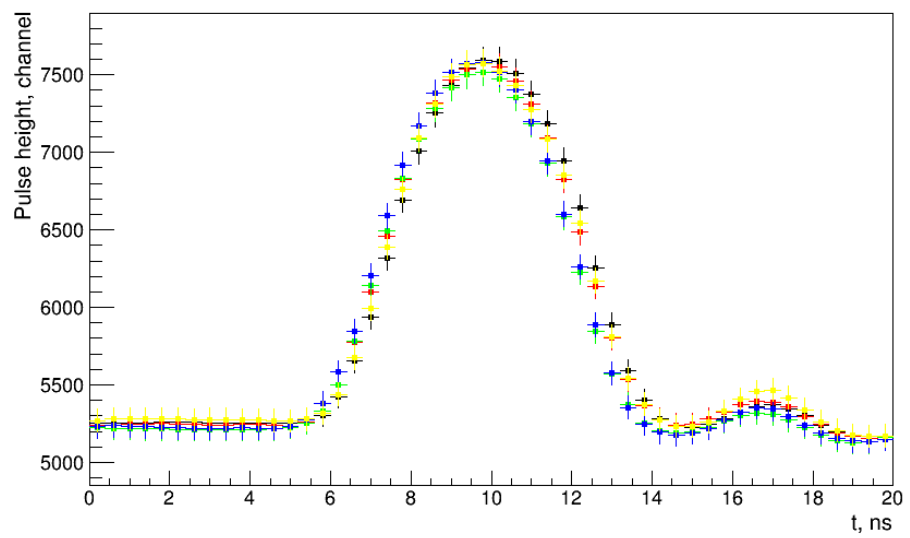

Fig. 4. Typical pulses of the $\mathrm{BC} 2$ detector measured with the $\mathrm{CAEN}$ digitizer.

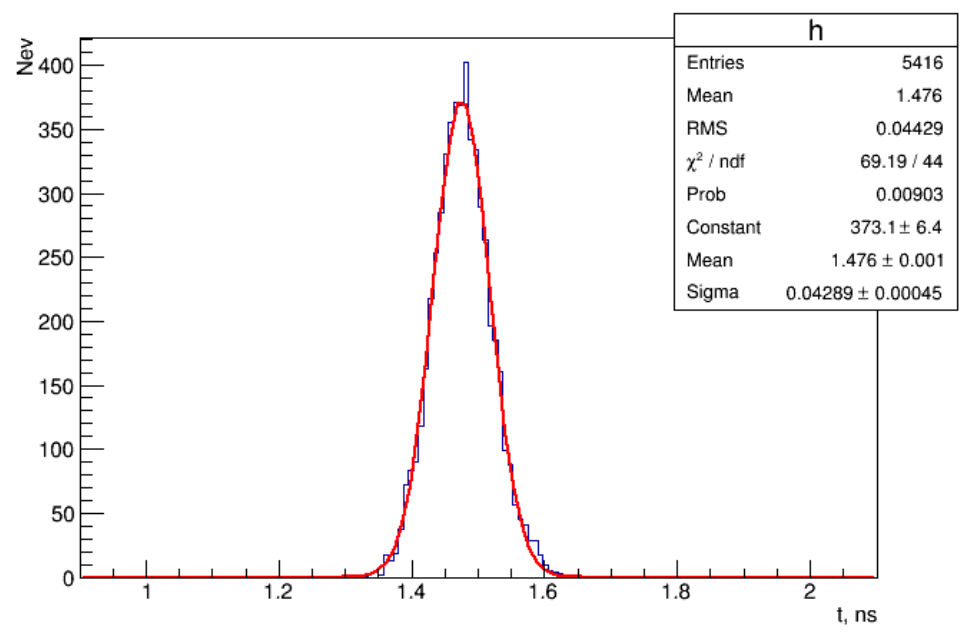

Fig. 5. The TOF peak obtained in measurement with Cherenkov and scintillation counters.

The active area of BD has a radius of $45 \mathrm{~mm}$ and a length of $150 \mathrm{~mm}$, consisting of 40 strips $\left(150 \times 7 \times 7 \mathrm{~mm}^{3}\right)$ made from a polished BC-418 scintillator wrapped in aluminized mylar. The scintillation light produced by charged particles is detected on single ends of the strips by $6 \times 6 \mathrm{~mm}$ Micro FC-60035-SMT SiPMs from Sensl. The detector pulses from the SiPMs are fed to the first-stage, low-noise amplifiers and then to the FEE board, which performs shaping, amplification, and discrimination functions. 
A time-over-threshold comparator is used in the discriminator producing LVDS pulses. A view of the strip assembly with SiPMs board is shown in Fig. 6.

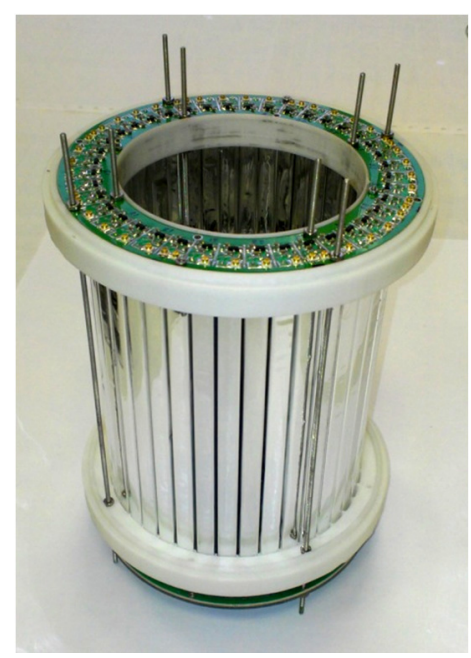

Fig. 6. A view of the strip assembly with the SiPMs board of the barrel detector.

A photo of the installed BD in the $2017 \mathrm{BM} @ \mathrm{~N}$ run with a $4.5 A \mathrm{GeV}$ beam of carbon ions is shown in Fig. 7. Typical analog pulses coming from a single FEE channel is shown in Fig. 8. The measurement was carried out with the CAEN digitizer. The BC3 detector and $\mathrm{SiD}$ were not installed in this run. The measurements showed that BD produced effective triggering of interactions of carbon ions with different targets, from $\mathrm{C}$ to $\mathrm{Pb}$.

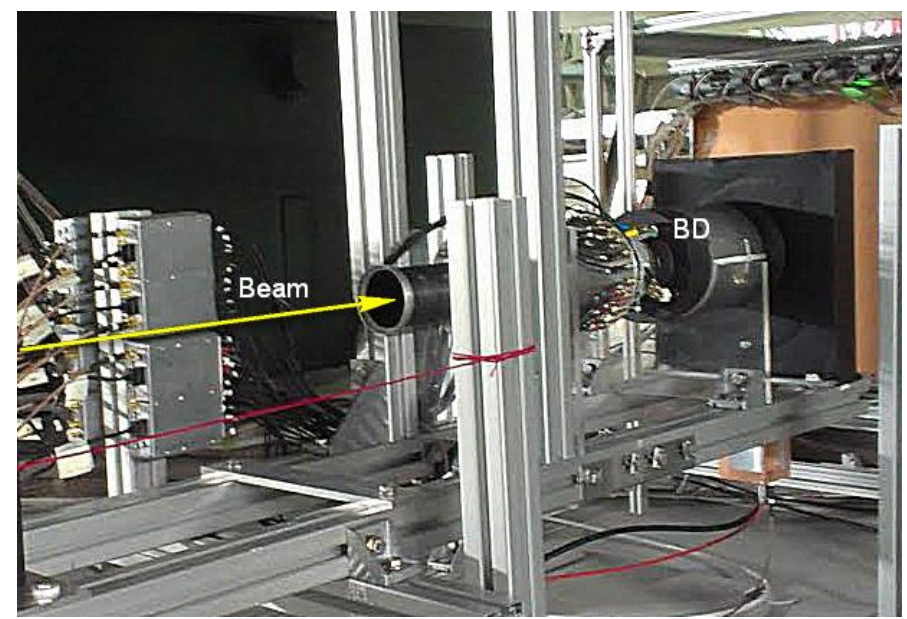

Fig. 7. A view of the barrel detector on the BM@N beam line (March 2017). 


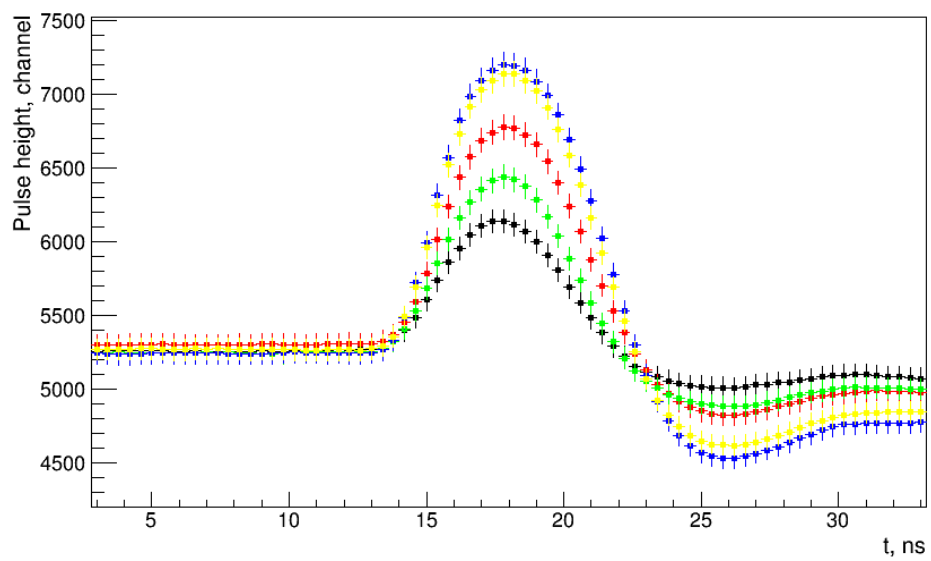

Fig. 8. Typical pulses of a single channel of the barrel detector.

\section{Conclusion}

The fast interaction trigger and T0 detectors, developed on a base of MCP-PMTs and SiPMs for the MPD and BM@N experiments at JINR, provide an efficient trigger of nucleus-nucleus collisions and the T0 pulses for TOF detectors with time resolution between 30 and 40 ps that corresponds to requirements.

The BM@N tests with the carbon-ion beam showed that beam detectors with thin plastic scintillators provide both the picosecond time resolution and good pulse height resolution for selection of beam ions in the trigger. In future runs with beams of highenergy nuclei, we plan to use scintillators with thickness between 0.15 and $1 \mathrm{~mm}$ in the beam counters.

On the basis of the obtained results and experience, we are making a final design of the FFD module and BM@N detectors. FFD module production, preparation of the target area detectors, and setup of the BM@N trigger for runs with beams of heavy nuclei from $\mathrm{Kr}$ to Au will take place in 2018.

\section{References}

1. V. Golovatyuk et al., Eur. Phys. J. A 52, 212 (2016).

2. M. Kapishin, Eur. Phys. J. A 52, 213 (2016).

3. V. Kekelidze et al., The NICA project at JINR Dubna, EPJ Web of Conferences 71, 00127 (2014).

4. V. I. Yurevich, O. I. Batenkov et al., Phys. Part. Nucl. Lett. 12, 778 (2015).

5. V. I. Yurevich and O. I. Batenkov, Nucl. Instrum. Meth. A 824, 162 (2016). 